\title{
Sang-Koo Woo $\cdot$ Hyun-Sik Kang \\ The apolipoprotein CIII T2854G variants are associated with postprandial triacylglycerol concentrations in normolipidemic Korean men
}

Received: 24 June 2003 / Accepted: 13 August 2003 / Published online: 27 September 2003

(C) The Japan Society of Human Genetics and Springer-Verlag 2003

\begin{abstract}
This study investigated associations between the apolipoprotein (apo) CIII polymorphism and triacylglycerol (TAG) concentrations in fasting and postprandial plasma. Polymerase chain reaction followed by a restriction fragment length genotyping was conducted to assess the allele frequency of the apo CIII T2854G variants in healthy and normolipidemic Korean men $(n=262)$. Waist circumference, body mass index (kilograms per meter squared), fasting plasma concentrations of TAG, total cholesterol, high-density lipoprotein cholesterol (HDLC), low-density lipoprotein cholesterol (LDLC), glucose, and insulin were compared across the genotypes. Compared to TT homozygotes and TG heterozygotes, GG homozygotes had $22 \%$ higher fasting TAG concentrations, respectively $(p<0.05)$. A subgroup of 60 subjects (TT homozygotes $=20$, TG heterozygotes $=22$, GG homozygotes $=18$ ) were further invited to participate in a high-fat meal test to assess postprandial TAG concentrations. During the high-fat meal test, the GG homozygotes had $21 \%$ higher TAG area under the curve (AUC) than the TT homozygotes $(p<0.05)$ and $22 \%$ higher TAG AUC than the TG heterozygotes $(p<0.05)$. In conclusion, this is the first study to show that the apo CIII T2854G variants are associated with elevated postprandial TAG concentrations in the study population of Korean men.
\end{abstract}

Keywords PCR-RFLP - apo CIII gene

polymorphism $\cdot$ Postprandial triacylglycerol

S.-K. Woo · H.-S. Kang $(\bowtie)$

School of Sport Science, SungKyunKwan University,

Natural Sciences Campus 300, Chunchun-dong,

Jangan-gu, Suwon, Kyunggi-do 440-746, South Korea

E-mail: hkang@skku.edu

Tel.: + 82-31-2996911

Fax: + 82-31-2996929

\section{Introduction}

Plasma apolipoprotein CIII (apo CIII), which consists of 79 amino acids, is synthesized predominantly in the liver and, to a lesser extent, in the intestine (Breslow 1995). Apo CIII is a major component of very-lowdensity lipoproteins (VLDL) and chylomicrons and a minor component of high-density lipoproteins (Breslow 1995).

While the functional role of the apo CIII is still unclear, it appears that apo CIII circulates in plasma with triglyceride-rich lipoproteins (TRL) and may interfere with lipolysis. For example, apo CIII inhibits lipoprotein lipase, a key enzyme hydrolyzing the triacylglycerol (TAG) portion of TRL, resulting in a delayed removal of TRL in vitro (Wang et al. 1985) and in vivo (Ginsberg et al. 1986). Apo CIII also interferes with apo E-mediated remnants removal by displacement of apo $\mathrm{E}$ from VLDL particles in vivo (Alto-Setala et al. 1996 and 1992).

The apo AI-CIII-AIV gene cluster, which is located in the long arm of chromosome 11 (Karathanasis 1985), has been known to have more than 20 polymorphic sites (Groenendijk et al. 2001). Among them, the SstI gene polymorphism located in the $3^{\prime}$ untranslated region of the apo CIII gene has been consistently implicated in elevated plasma TAG concentrations in several populations such as Caucasians (Paul-Hayase et al. 1992; Stocks et al. 1987; Dammerman et al. 1993) and nonCaucasians (Zeng et al. 1995; Hong et al. 1997; Henderson et al. 1987). In addition, the rare allele of the $\mathrm{T} 2854 \mathrm{G}$ polymorphism located in the intergenic region of the apo CIII gene was recently reported to be associated with elevated fasting (Minihane et al. 2002) or postprandial TAG concentrations (Waterworth et al. 1999) in Caucasians. However, little information on the influence of this gene polymorphism on plasma lipids, especially in a postprandial state, is currently available in non-Caucasians such as Koreans. Therefore, the purpose of the current study was to investigate possible 
associations between the apo CIII T2854G variants and fasting lipids and postprandial TAG concentrations in healthy and normolipidemic Korean men.

\section{Materials and methods}

\section{Subjects}

Apparently healthy and normolipidemic Korean men $(n=262$ ages 26.6-33.0 years) who had no current major diseases and no family history of coronary heart diseases, were recruited to participate in the study. Participants signed a written consent approved by the Institutional Human Subject Review Board. None was taking medications or hormones affecting lipid and lipoprotein metabolism. No smoking and alcohol consumption were allowed for $24 \mathrm{~h}$ prior to the experiment. Weight was assessed with a balance scale and height with a stadiometer; body mass index (BMI) was calculated (kilograms per meter squared). Waist circumference was measured at the umbilicus level. Following an overnight fast, venous blood samples from an antecubital vein were collected into potassium EDTA tubes for genomic DNA extraction and fasting lipid measurements.

\section{Genotyping}

Genomic DNA was extracted from plasma buffy coats using QiaAmp DNA Blood Mini Kit (Qiagen, CA, USA). The extracted DNA was stored at $-80^{\circ} \mathrm{C}$ until analysis. The $\mathrm{T} 2854 \mathrm{G}$ allele variant of the apo CIII gene was determined using polymerase chain reaction-restriction fragment length polymorphism (PCR-RFLP), as previously described (Waterworth et al. 1999), with modifications.

In brief, polymerase chain reaction was performed in a GeneAmp PCR system 9700 (Applied Biosystems, Foster City, CA, USA) with a $20-\mu 1$ reaction volume containing $30-50 \mathrm{ng}$ of genomic DNA, 20 pmol of each primer, and $1 \times$ Taq PCR master mix (Qiagen, Valencia, CA, USA). The Taq PCR master mix provides a final concentration of $2.5 \mathrm{U}$ of Taq DNA polymerase, PCR buffer $(1.5 \mathrm{mM} \mathrm{MgCl} 2)$, and $200 \mu$ each dNTPs. PCR conditions were as follows: initial denaturation at $94^{\circ} \mathrm{C}$ for $5 \mathrm{~min}$, followed by 35 cycles of denaturation at $94^{\circ} \mathrm{C}$ for $30 \mathrm{~s}$, annealing at $65^{\circ} \mathrm{C}$ for $30 \mathrm{~s}$, and extension at $72^{\circ} \mathrm{C}$ for $30 \mathrm{~s}$, and final extension at $72^{\circ} \mathrm{C}$ for $7 \mathrm{~min}$. The primer sequences were as follows: forward $5^{\prime}$-CAA CAG GAG CTG TCC TTC AGT TCT GCC - $3^{\prime}$ and reverse 5'GGT CAG TCC AGA GGT CAG AGT CAG GAG GAG-3'.

The PCR products were digested with the restriction enzyme Alw26I (MBI Fermentas Inc, Hanover, MD, USA), separated by electrophoresis using low melting temperature $2.5 \%$ agarose gel (ICN Biomedicals Inc, Aurora, OH, USA) and visualized by ethidium bromide staining (Sigma, St. Louis, MO, USA). The rare allele was detected by the presence of a cutting site. Genotyping was successfully completed for all the 262 subjects.

\section{High-fat meal test}

A subgroup of 60 subjects (TT homozygotes $=20$, TG heterozygotes $=22$, and GG homozygotes $=18$ ) was further invited to participate in postprandial TAG response to a high-fat meal. No exercise was permitted $48 \mathrm{~h}$ before the test, and no alcohol or smoking was allowed for $24 \mathrm{~h}$ before the test. Following an overnight fast, subjects arrived at the Clinical Laboratory Unit. An indwelling cannula was inserted into the antecubital vein of the forearm and a fasting sample was taken $(0 \mathrm{~h})$. After consumption of the standard fat meal consisting of $22 \mathrm{~g}$ dairy milk protein, $56 \mathrm{~g}$ carbohydrates, $66 \mathrm{~g}$ dairy milk fat (of which $42 \mathrm{~g}$ was saturated), and $417 \mathrm{mg}$ cholesterol, venous blood samples were collected at 2 , 3 , 4 , and $6 \mathrm{~h}$ to assess postprandial TAG concentrations.
Postprandial TAG during the high-fat meal test was expressed as area under the curve (AUC) calculated by the trapezoidal rule.

Fasting lipid, glucose, and insulin measurements

All blood samples were collected into potassium EDTA tubes. Samples were separated by centrifugation at $4,600 \mathrm{~g}$ for $10 \mathrm{~min}$ and the plasma was removed and stored at $-80^{\circ} \mathrm{C}$ until analysis. Total cholesterol and TAG concentrations were measured by enzymatic colorimetry methods with commercial kits (Boehringer Mannheim, Mannheim, Germany). High-density lipoprotein cholesterol (HDLC) concentration was determined by measuring cholesterol in the supernatant after precipitation of apo B-containing lipoproteins with magnesium chloride and dextran sulfate (Sigma, St. Louis, USA). Low-density lipoprotein cholesterol (LDLC) concentration was calculated using the Friedewald formula (1972). Fasting glucose was determined by an enzymatic method (Beckman Diagnostics, Fullerton, CA, USA), and fasting insulin concentration was determined using the IMx Insulin Assay (Abbott Labs, Abbott Park, IL, USA).

\section{Statistical analyses}

The statistical program used for the analyses was SPSS-PC version 10.1. The distributions of the dependent variables were checked for normality prior to the analyses by plotting standardized residuals for each variable from regression models using age as an independent variable. Because TAG was not normally distributed, a $\log _{10}$ transformation was performed for statistical analyses.

Results were expressed as mean \pm SD. Allelic frequencies were estimated by the genotype frequencies. Univariate analysis of variance followed by Bonferroni test was used to compare measured fasting lipids among the genotypes. The postprandial response was compared among the genotypes by univariate analysis of variance followed by Bonferroni test (1) for each time point TAG and (2) for AUC TAG. Statistical significance was tested at $p=0.05$.

\section{Results}

Figure 1 illustrates the examples of the apo CIII T2854G variants. Table 1 represents the allele frequencies of the $\mathrm{T} 2854 \mathrm{G}$ variants. The frequency of the rare allele $\mathrm{G}$ was 0.37 , which was comparable with those of Caucasians (Minihane et al. 2002; Waterworth et al. 1999).

Table 2 represents physical and laboratory profiles of the participants according to the genotypes. There were no significant differences in average age, BMI, and waist circumference among the genotypes. With respect to fasting plasma, however, the finding that the GG homozygotes had $22 \%$ higher TAG concentrations than TT homozygotes $(p<0.05)$ and the TG heterozygotes $(p<0.05)$ was similar to those of previous studies. In addition, the further analyses classified by BMI ( $>25$ or $<25$ ) showed that compared to either the TT homozygotes or the TG heterozygotes, the GG homozygotes had still significantly elevated fasting TAG concentrations independent of the dichotomy (data not shown).

Table 3 represents the physical and laboratory profiles of the subgroup who participated in the high-fat meal test. With respect to the postprandial TAG concentrations, it was of particularly interest to find that the GG 
Fig. 1 Examples of the apo CIII T2854G variants. Lanes 1 , 18, 33: 25-bp size markers. Lanes 7, 14, 21, 23, 32: TT homozygotes. Lanes $2,4,5$, $8-13,15,17,20,22,24,25$, 27-29, 31: TG heterozygotes. Lanes 3, 6, 16, 19, 26, 30: GG homozygotes

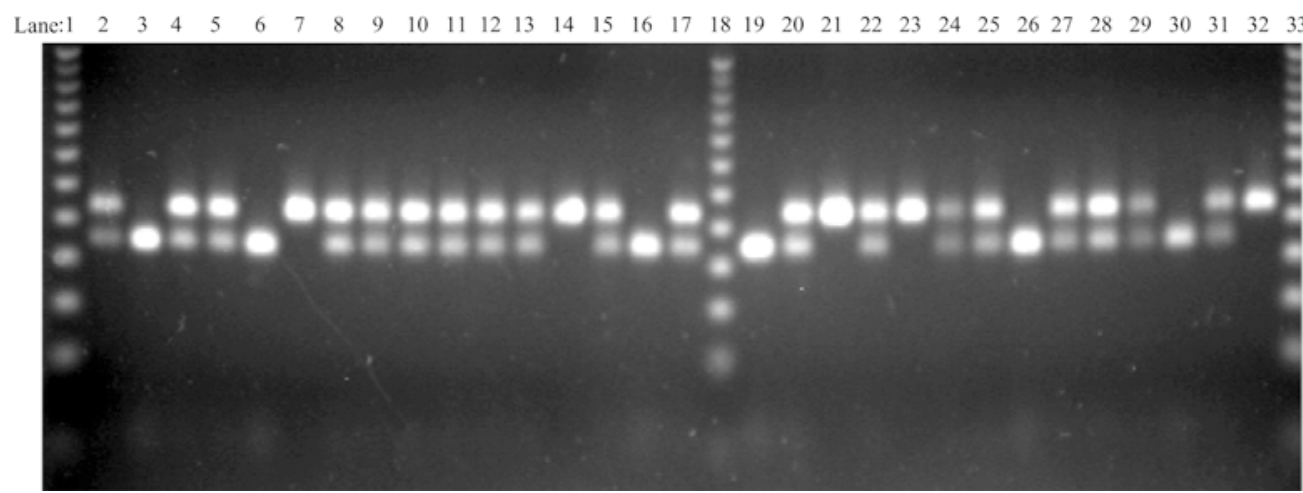

Table 1 The genotype and allele frequencies of the apolipoprotein (apo) CIII T2854G variants

\begin{tabular}{llll}
\hline $\begin{array}{l}\text { Genotype/ } \\
\text { allele }\end{array}$ & $\begin{array}{l}\text { Current } \\
\text { study }\end{array}$ & $\begin{array}{l}\text { Minihae et al. } \\
2002\end{array}$ & $\begin{array}{l}\text { Waterworth et al. } \\
1999\end{array}$ \\
\hline TT & $99(0.38)$ & $55(0.41)$ & $284(0.40)$ \\
TG & $133(0.51)$ & $62(0.46)$ & $348(0.49)$ \\
GG & $30(0.11)$ & $18(0.13)$ & $85(0.11)$ \\
T & $166(0.63)$ & $86(0.64)$ & $458(0.64)$ \\
G & $96(0.37)$ & $49(0.36)$ & $259(0.36)$ \\
Total & 262 & 135 & 717 \\
\hline
\end{tabular}

homozygotes had $21 \%$ higher TAG AUC than the TT homozygotes $(p<0.05)$ and $22 \%$ higher TAG AUC than the TG heterozygotes $(p<0.05)$ during the test. The plot of the test according to the genotypes is shown in Fig. 2. In addition, there was a tendency for the GG homozygotes to have higher TG and waist circumference than the TT homozygotes or the TG heterozygotes, but neither TG nor waist circumference was significantly different across the genotypes, as shown in Table 3.

\section{Discussion}

While the rare alleles of XmnI and SstI polymorphic sites within the apo AI-CIII-AIV complex were previ- ously associated with increased TAG concentrations in Korean hypertriglyceridemic subjects (Hong et al. 1997), this is the first study to report that the GG homozygotes of the apo CIII T2854G gene polymorphism may be implicated in elevated postprandial TAG concentrations during a high-fat meal test.

The finding - that compared to the TT homozygotes and the TG heterozygotes, the GG homozygotes had significantly higher TAG AUC during the high-fat meal test - suggests that the G allele may have an impact on TAG metabolism via an impaired lipolysis and thereby TAG concentrations in circulating fasting plasma. Therefore, it is speculated that the polymorphic site itself, due to its intergenic location, may be functionally important in postprandial lipolysis (Minihane et al. 2002; Waterworth et al. 1999). Second, it is possible that the polymorphic impact modulates plasma TAG concentrations by linkage disequilibrium with other functional sequences in the apo AI-CIII-AIV gene cluster (Waterworth et al. 1999). Third, there was a tendency for the GG homozygotes to have a relatively larger waist circumference than the TT homozygotes or the TG heterozygotes, suggesting a possible interaction between the rare allele and central obesity in TAG metabolism and thereby its concentrations in circulating plasma. However, this possible interaction between this polymorphism and abdominal obesity is yet to be established in a large sample.

Table 2 Comparisons of anthropometric and laboratory measures across the genotypes, adjusted for age and body mass index (BMI) $(n=262)$. TAG triacylglycerol, Waist waist circumference, $A U C$ area under the curve, $C I$ confidence interval. $H D L C$ high-density lipoprotein cholesterol, $L D L C$ low-density lipoprotein cholesterol

\begin{tabular}{lccrr}
\hline Variables & \multicolumn{2}{l}{ Genotype } & & 95\% CI \\
\cline { 2 - 4 } & TT & TG & GG & \\
\hline Age, year & $29.7 \pm 1.5$ & $30.2 \pm 1.4$ & $30.0 \pm 1.2$ & $29.8-30.2$ \\
Waist, cm & $82.8 \pm 15.1$ & $79.9 \pm 16.1$ & $83.5 \pm 12.7$ & $79.2-83.0$ \\
BMI, kg/m & $25.5 \pm 7.2$ & $24.0 \pm 7.3$ & $26.3 \pm 6.9$ & $23.7-25.5$ \\
*TAG, mM & $1.18 \pm 0.40$ & $1.19 \pm 0.46$ & $1.52 \pm 0.64$ & $1.16-1.28$ \\
TC, mM & $4.00 \pm 0.80$ & $3.92 \pm 0.71$ & $4.15 \pm 0.91$ & $3.87-4.06$ \\
HDLC, mM & $1.18 \pm 0.26$ & $1.18 \pm 0.26$ & $1.07 \pm 0.25$ & 0.143 \\
LDLC, mM & $2.52 \pm 0.73$ & $2.56 \pm 0.97$ & $2.69 \pm 0.80$ & 0.001 \\
Glucose, mM & $5.06 \pm 0.37$ & $5.13 \pm 0.40$ & $5.08 \pm 0.36$ & 0.452 \\
Insulin, pM & $107.48 \pm 61.70$ & $103.31 \pm 49.59$ & $128.06 \pm 43.42$ & $5.01-5.12$ \\
\hline
\end{tabular}

*TAG was $\log _{10}$-transformed for statistical analysis, but actual means and SD are presented

For TAG, GG is significantly different from TT $(p<0.05)$ and TG $(p<0.05)$ 
Table 3 Comparison of physical and biochemical measures across the subgroup participating in the high-fat meal test $(n=60)$. Waist waist circumference, $B M I$ body mass index, TAG triacylglycerol, $A U C$ area under the curve, $C I$ confidence interval, $H D L C$ high-density lipoprotein cholesterol, $L D L C$ low-density lipoprotein cholesterol

\begin{tabular}{lccrr}
\hline Variables & \multicolumn{2}{l}{ Genotype } & & 95\% CI \\
\cline { 2 - 4 } & TT $(n=20)$ & TG $(n=22)$ & GG $(n=18)$ & \\
\hline Age, year & $30.7 \pm 0.9$ & $31.3 \pm 1.2$ & $30.8 \pm 3.3$ & $30.4-31.4$ \\
Waist, cm & $83.7 \pm 11.4$ & $84.0 \pm 14.6$ & $89.1 \pm 6.7$ & $82.4-88.5$ \\
BMI, $\mathrm{kg} / \mathrm{m}^{2}$ & $23.8 \pm 4.4$ & $24.1 \pm 5.4$ & $25.7 \pm 4.0$ & $23.7-25.7$ \\
TAG, mM & $1.43 \pm 0.09$ & $1.44 \pm 0.11$ & $1.51 \pm 0.13$ & 0.556 \\
*AUC, mM/h & $3.84 \pm 0.91$ & $3.81 \pm 0.77$ & $4.65 \pm 1.48$ & $3.79-4.36$ \\
TC, mM & $4.24 \pm 0.76$ & $4.45 \pm 0.81$ & $4.40 \pm 0.64$ & $4.17-4.56$ \\
HDLC, mM & $1.21 \pm 0.30$ & $1.17 \pm 0.20$ & $1.09 \pm 0.28$ & 0.418 \\
LDLC, mM & $2.38 \pm 0.61$ & $2.60 \pm 0.74$ & $2.51 \pm 0.71$ & 0.028 \\
Glucose, mM & $5.06 \pm 0.40$ & $5.09 \pm 0.33$ & $5.09 \pm 0.37$ & 0.659 \\
Insulin, $\mathrm{pM}$ & $116.38 \pm 76.07$ & $112.79 \pm 59.91$ & $132.89 \pm 42.98$ & $4.93-2.68$ \\
\hline
\end{tabular}

*For AUC, GG is significantly different from TT $(p<0.05)$ and TG $(p<0.05)$

Fig. 2 Postprandial triacylglycerol concentrations across the genotypes

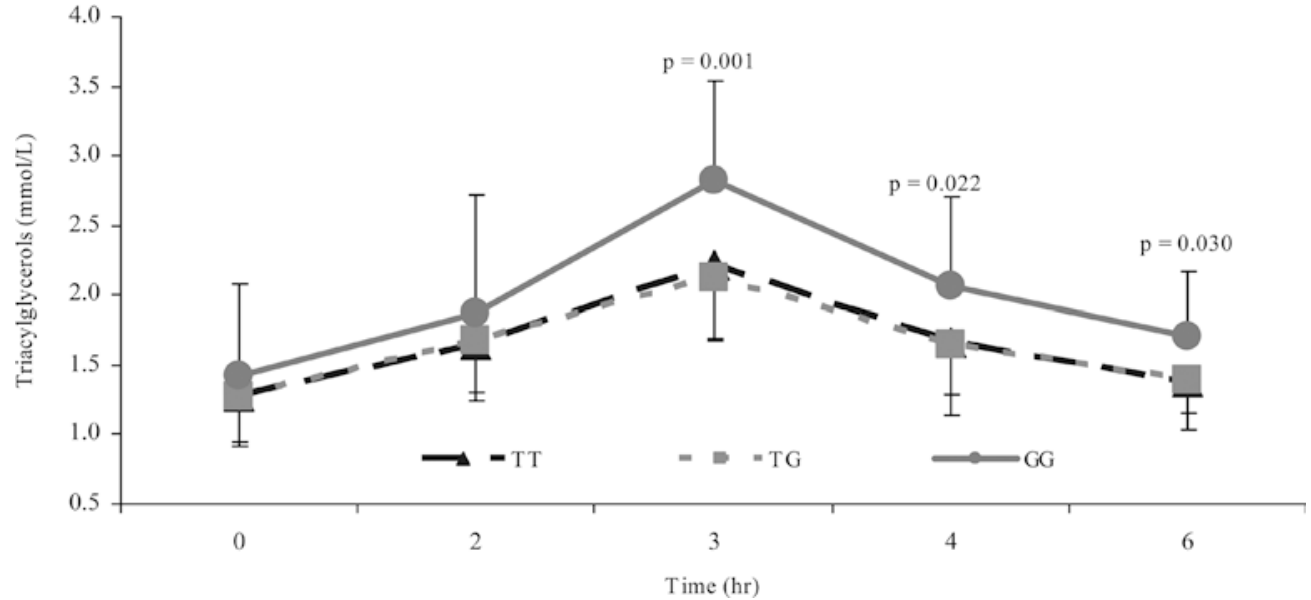

Although the rare allele of the apo CIII T2854G gene polymorphism was associated with elevated TAG concentrations in the fasting and postprandial state in this study population, caution is necessary because the physiologic significance of the mutation has yet to be examined. First, while the rare allele (S2) of the apo AICIII-AIV complex was positively associated with apo CIII and TAG concentrations in healthy individuals (Shoulders et al. 1991), whether or not a similar correlation between the T2854G polymorphism and plasma apo CIII concentrations exists still needs to be investigated in future studies.

In summary, postprandial hyperlipemia has been complicated in the metabolic syndrome (Gott Jr 1998; Jeppesen et al. 1995) consisting of visceral obesity, hyperlipemia, insulin resistance, glucose intolerance, and hypertension, which collectively increases the risk of cardiovascular disease (CVD). In the currently study, we report that the GG homozygotes of the apo CIII T2854G gene polymorphism had elevated TAG concentrations not only in the fasting but also in the postprandial state in the study population, implying a potential contribution of this polymorphism to the variation in susceptibility to the CVD.

\section{References}

Aalto-Setala K, Fisher EA, Chen X, Chajek-Shaul T, Hayek T, Zechner R, Walsh A, Ramakrishnan R, Ginsberg HN, Breslow JL (1992) Mechanism of hypertriglyceridemia in human apolipoprotein (apo) C-III transgenic mice. Diminished very low density lipoprotein fractional catabolic rate associated with increased apo C-III and reduced apo E on the particles. J Clin Invest 90: 1889-1900

Aalto-Setala K, Weinstock PH, Bisgaier CL, Wu L, Smith JD, Breslow JL (1996) Further characterization of the metabolic properties of triglyceride-rich lipoproteins from human and mouse transgenic mice. J Lipid Res 37: 1802-1811

Breslow JL (1995) Familial disorders of high density lipoprotein metabolism. In: Scriver CR, Beaudet Al, Sly WS, Valle D (eds) The metabolic basis of inherited disease. McGraw-Hill, New York, pp 1251-1266

Dammerman M, Sandkuijl LA, Hallas JL, Chung W, Breslow JL (1993) An apolipoprotein CIII haplotype protective against hypertriglyceridemia is specified by promoter and 3' untranslated region polymorphisms. Proc Natl Acad Sci USA 90: $4562-4566$

Friedewald WT, Levy RI, Fredrickson DS (1972) Estimation of the concentration of low-density lipoprotein cholesterol in plasma, without use of the preparative ultracentrifuge. Clin Chem 18: 499-502

Ginsberg HN, Le N-A, Goldberg IJ, Gibson JC, Rubinstein A, Wang-Iverson P, Norum R, Brown WV (1986) Apolipoprotein 
B metabolism in subjects with deficiency of apolipoproteins CIII and AI: Evidence that apolipoprotein CIII inhibits catabolism of triglyceride-rich lipoproteins by lipoprotein lipase in vivo. J Clin Invest 78: 1287-1295

Gott Jr AM (1998) Triglycerides as a risk factor for coronary artery disease. Am J Cardiol 82:22Q-25Q

Groenendijk M, Cantor RM, de Bruin TWA, Dallinga-Thie GM (2001) The apoAI-CIII-AIV gene cluster. Atherosclerosis 157: $1-11$

Henderson HE, Landen SV, Michie J, Berger GMB (1987) Association of DNA polymorphism in the apolipoprotein CIII gene with diverse hyperlipidaemic phenotypes. Hum Genet 75: 62-65

Hong SH, Park WH, Lee CC, Song JH, Kim JQ (1997) Association between genetic variations of apo AI-CIII-AIV cluster gene and hypertriglyceridemia subjects. Clin Chem 43: 13-17

Jeppesen J, Hollenbeck CB, Zhou M-Y, Coulston Am, Jones C, Chen YD, Reaven GM (1995) Relation between insulin resistance, hyperinsulinemia, postpheparin plasma lipoprotein lipase activity, and postprandial lipemia. Arteriosclero Thromb Vasc Biol 15:320-324

Karathanasis SK (1985) Apolipoprotein multigene family: Tandem organization of human apolipoprotein Ai, CIII, and AIV genes. Proc Natl Acad Sci USA 82: 6374-6378

Minihane AM FY, Talmud P, Leigh-Firbank EC, Williams CM (2002) Influence of the APOC3-2854T $>\mathrm{G}$ polymorphism on plasma lipid levels: effect of age and gender. Biochimica et Biophysica Acta 1583: 311-314
Paul-Hayase H, Rosseneu M, Robinson D, van Bervliet JP, Deslypere JP, Humphries SE (1992) Polymorphisms in the apolipoprotein (apo) AI-CIII-AIV gene cluster: detection of genetic variation determining plasma apo AI, apo CIII and apo AIV concentrations. Hum Genet 88: 439-446

Shoulders CC, Harry PJ, Lagrost L, White SE, Shah NF, North JD, Gilligan M, Gambert P, Ball MJ (1991) Variation at the apo AI/CIII/AIV gene complex is associated with elevated plasma levels of apo CIII. Arterosclerosis 87: 239-247

Stocks J, Paul-Hayase H, Galton DJ (1987) Haplotypes identified by DNA restriction-fragment-length polymorphisms in the AIC-III-A-IV gene region and hypertriglyceridemia. Am J Hum Genet 41: 106-118

Wang CS, McConathy WJ, Kloer HU, Alaupovic P (1985) Modulation of lipoprotein lipase activity by apolipoproteins. Effect of apolipoprotein C-III. J Clin Invest 75: 384-390

Waterworth DM, Ribalta J, Nicaud V, Dallongeville J, Humphries SE, Talmud P; on the behalf of the EARS group (1999) ApoCIII gene variants modulate postprandial response to both glucose and fat tolerance tests. Circulation 99: 1872-1877

Zeng Q, Dammerman M, Takada Y, Matsunaga A, Breslow JL, Sasaki J (1995) An apolipoprotein CIII marker associated with hypertriglyceridemia in Caucasians also confers increased risk in a west Japanese population. Hum Genet 95: 371-375 